\title{
Texture Segmentation using LBP embedded Region Competition
}

\author{
Xu Qing, Yang Jie, Ding Siyi \\ Inst. of Image Processing \& Pattern Recognition, Shanghai Jiao tong Univ. Shanghai BOX251 1954 HUASHAN \\ ROAD, SHANGHAI, P.R.China
}

\begin{abstract}
In this paper, we modify the region competition method to segment textures. First, local Binary pattern (LBP) histogram is adopted to capture the texture information. Then, considering the specific goal of texture segmentation, we propose new assumption about region competition and rewrite the energy function based on LBP histograms. We also develop the two-stage iterative algorithm to make our energy converge to a local minimum. Because of the fast LBP operator and nonparametric histogram model, we can simplify the step of parameter estimating, which is always the most time-consuming. Besides, LBP`s high performance for texture characterization helps to make our method more suitable for texture segmentation problem. Experiments show that the performance of our proposed method is promising, and a robust and fast segmentation of texture images is obtained.
\end{abstract}

Key words: region competition, local binary pattern

\section{Introduction}

Texture segmentation plays an important part in low level image analysis and understanding. Basically, it involves the identification of regions with the same texture features, so that further analysis can be performed on the respective regions alone. An effective segmentation algorithm is very useful in many areas, such as analysis of remote sensing images, industrial monitoring of product quality, medical image analysis, and image retrieval.

Zhu and Yuille [1] proposed a statistical and variational framework for image segmentation, which is named Region Competition. Their algorithm is derived by minimizing a generalized Bayes/MDL criterion using the variational principle and the global energy is guaranteed to converge to a local minimum. It

Correspondence to: qingxu8@hotmail.com

Recommended for acceptance by Xavier Otazu

ELCVIA ISSN: 1577-5097

Published by Computer Vision Center / Universitat Autonoma de Barcelona, Barcelona, Spain 
combines the attractive aspects of both active contours and region growing to achieve segmentation with regular boundary. Due to its effectiveness, region competition has been widely adopted in medical image series and video series segmentation [2 34 4]. However, application to texture segmentation may need some modification. First, in order to split an image into sub regions with homogenous texture features, region competition must employ a highly discriminating texture feature rather than intensity to represent a pixel. Zhu and Yuille [1] have extended their algorithm to texture segmentation by using the gradient as the feature. But this feature can not capture efficiently the local pattern of texture elements. Second, region competition must require an assumed texture model and its model parameters need to estimate in every round of greedy algorithm. So we must avoid using complex models, whose parameters are difficult to estimate.

In this paper, we choose local binary pattern (LBP) [5] operator as texture feature descriptor. LBP operator codes a local window pattern from a texture patch, and its histogram is often treated as texture feature in classification problem. Recent studies have shown that excellent texture discrimination can be obtained with local binary patterns (LBP) [6] [7]. In order to obtain a robust segmentation, we employ LBP histogram of a local circular window to represent the feature of the center pixel. The LBP distribution of one region is also approximated by a LBP histogram. Thus we reassume that our goal of texture segmentation is to divide an image into sub regions, where each local LBP histogram is similar to the global LBP histogram. Based on that assumption, we propose a new energy definition and redevelop the two-stage greedy algorithm to converge our energy to a local minimum. Because LBP histograms are computed with low complexity, we actually simplify the complex estimating of assumed model parameters and thus enhance the efficiency of region competition.

This paper is organized as follows. First, we will review region competition algorithm in section 2 and also give a brief introduction to LBP in section 3. Then, we proposed our region competition based on LBP histogram in section 4, where a new energy function is proposed and the greedy algorithm is also redeveloped. The simulation results and discussions are presented in section 5. Finally, conclusions are drawn in Section 6.

\section{Region competition for gray level image}

Zhu and Yuille [1] have proposed a hybrid statistical segmentation framework, called region competition. Then they applied this method to gray level image segmentation and obtained a promising result. In this section, this approach is briefly reviewed.

The goal of region competition is to split images into homogenous sub regions. Zhu and Yuille has assumed that a region $R$ is considered to be homogenous only if its intensity values are generated by one of a family of specific probability distributions $P(I \mid \alpha)$, where $\alpha$ denotes the model parameters of that distribution and $I$ represents the intensity value. We express the energy function as follows:

$$
E\left(\Gamma,\left\{\alpha_{i}\right\}\right)=\sum_{i=1}^{M}\left\{\frac{\mu}{2} \int_{\partial R_{i}} d s-\sum_{(x, y) \in R_{i}} \log P\left(I_{(x, y)} \mid \alpha_{i}\right)+\lambda\right\},
$$

where $\Gamma$ is the boundary in the image, $P(\bullet)$ is a specific distribution for region $R_{i}, \alpha_{i}$ is its parameters, $\mathrm{M}$ is the number of the regions, and $\mu$ and $\lambda$ are two constants. $I_{(x, y)}$ is intensity value for pixel $(x, y)$. So it is sensible to minimize this energy function in order to split an image into homogenous sub regions.

Region competition starts by initializing the segmented image with several randomly selected seeds. All these seeds are considered to be different regions while all background not covered by seeds are treated as one single region. Then a two stage greedy algorithm is employed to converge the global energy $E$ to a local minimum. The first stage minimizes the energy with the number of regions fixed, and the second merges the pair of regions if this merging can decrease the global energy. The two stages are performed iteratively until the global energy $E$ converges to a local minimum. Moreover, the first stage is also divided into two steps. Firstly, the boundary $\Gamma$ is fixed and the parameters $\alpha_{i}$ for different regions ${ }^{R_{i}}$ are estimated using a maximum 
likelihood criterion. Then we fix ${ }^{\alpha_{i}}$, and thus $P\left(I_{(x, y)} \mid \alpha_{i}\right)$ becomes the conditional probability distribution. The boundary is moved along the deepest decent in order to minimize the energy function. If there are some image regions that are not occupied by any regions, new seeds will be put into such regions to execute the above two stages alternatively again

However, it is inappropriate to directly apply the above method to texture segmentation. Firstly, we should notice the assumption about the homogenous regions in region competition. Actually the simply intensity distributions sometimes fail to capture all the information about a texture. Textures with the same intensity distribution can be different textures due to different spatial arrangement of intensity values. So we must employ a discriminating texture feature, which can characterize not only the intensity distributions but also the spatial pattern. Secondly, the above method has indicated that the forms of the underlying feature value distribution have to be assumed in advance. We must assume a texture model first, and then estimate its parameters in each round of greedy algorithms. Unfortunately most of texture models are very complex and estimating their parameters is very time-consuming, even sometimes involving training. Therefore, a simple model is preferred in our work to describe the feature distributions.

\section{Texture description using local binary pattern}

The texture content of a region can be characterized [5] by the distribution of Local binary pattern (LBP). In this section, we will give a brief introduction to the original LBP operator and its extensions.

The LBP operator was first introduced as a complementary measure for local image contrast [8]. This operator thresholds eight neighbors [Fig.1.a] of a pixel with the value of that center pixel [Fig.1.b]. Then the LBP code [Fig.1.d] are computed by multiplying the thresholded values by the binomial weights given to the corresponding pixels [Fig.1. c] and summing up the results.

\begin{tabular}{|l|l|l|}
\hline 17 & 15 & 24 \\
\hline 12 & 23 & 39 \\
\hline 14 & 13 & 40 \\
\hline
\end{tabular}

(a)

\begin{tabular}{|c|c|c||c|c|c||c|c|c|}
\hline 0 & 0 & 1 & 1 & 2 & 4 & 0 & 0 & 4 \\
\hline 0 & & 1 & 128 & & 8 & 0 & & 8 \\
\hline 0 & 0 & 1 & 64 & 32 & 16 & 0 & 0 & 16 \\
\hline
\end{tabular}

LBP $=4+8+16=28$

Fig. 1. Computation of local binary pattern (LBP)

Although LBP captures the spatial pattern of local texture, it fails to characterize the contrast of the texture. So LBP can be improved by taking into account the local contrast for a 3x3 neighbours. The local contrast $\mathrm{C}$ is calculated by subtracting the average of the gray levels below the center pixel from that above the center pixel. So the joint distribution of LBP and contrast $C$ is used as texture features. Because it is difficult to find a general parametric model for this joint distribution, it is approximated by a two dimensional discrete histogram. Having made a trade-off between discrimination and stability of histograms, we quantize the contrast using a uniform quantizer with 8 quantization levels. So the size of the histogram is 256x8.

Using LBP/C, very good discrimination rates were reported with textures selected from the photographic album of Brodatz [5]. LBP/C operator also outperforms many other methods in terms of computation complexity, which makes it suitable for the image retrieval task [5]. Moreover, we can extend LBP/C to characterize color texture simply by quantizing the color features first [9]. So we embed the region competition framework with LBP/C feature to segment texture and even color texture. 


\section{Texture segmentation with region competition}

The LBP code on a pixel can not describe its texture information completely, since texture is often defined on a local patch. We place a circular window around a pixel, and the feature of a pixel is characterized by the LBP histogram of that window. The larger the window, the more likely the window is representative of the texture distribution and thus the smaller misclassification. But a larger window may also make us unable to locate the boundary accurately. A region can also be modeled by a LBP histogram. The goal of our segmentation is to split an image into homogenous texture regions. In each of these regions, we assume the LBP distribution of arbitrary local circular window is similar to the global LBP distribution of the region. This assumption is different from the one for gray image segmentation---each homogenous region comes from a certain stochastic process. Thus we rewrite the global energy function according to that assumption as:

$$
E\left(\Gamma,\left\{\alpha_{i}\right\}\right)=\sum_{i=1}^{M}\left\{\frac{\mu}{2} \int_{\partial R_{i}} d s-\sum_{(x, y) \in R_{i}} S\left(H_{(x, y)}, H_{i}\right)+\lambda\right\},
$$

where denotes $H_{(x, y)}$ the LBP histogram for the local circular window around pixel $(x, y)$, and $H_{i}$ is the LBP histogram for the region $R_{i} \cdot S\left(H_{(x, y)}, H_{i}\right)$ represents the similarity between $H_{(x, y)}$ and $H_{i}$.

The similarity measure $S\left(H_{1}, H_{2}\right)$ of two histograms is defined by

(3)

$$
S\left(H_{1}, H_{2}\right)=1-\sum_{i=1}^{k}\left(H_{1}(i)-H_{2}(i)\right)^{2},
$$

where $\mathrm{k}$ is the size of histogram bins. The closer $S\left(H_{1}, H_{2}\right)$ is to 1 , the more alike two histogram.

Because the energy in equation (2) depends on two factors-including the segmentation $\Gamma$ and the histograms $H_{i}$ for each region $R_{i}$-we adopt the greedy algorithm, consisting of two stages. In the first stage, the number of regions is fixed and the boundary is moved to minimize the energy function. In the second stage, the neighboring regions are merged if this merging can decrease the energy function. The algorithm proceeds until the energy converge into a local minimum.

We will develop the first stage in detail.

In the first step, we fix the boundary $\Gamma$ to minimize the energy function. In other words, each region ${ }_{R_{i}}$ is fixed and we solve $H_{i}$ to minimize the description cost for $R_{i}$.

$$
\begin{aligned}
H_{i}^{*} & =\arg \min _{H_{i}}\left(-\sum_{(x, y) \in R_{i}} S\left(H_{(x, y)}, H_{i}\right)\right) \\
& =\arg \min _{H_{i}}\left(\sum_{(x, y) \in R_{i}} \sum_{m=1}^{k}\left(H_{(x, y)}-H_{i}\right)^{2}\right) \\
& =\frac{1}{N} \sum_{(x, y) \in R_{i}} H_{(x, y)}
\end{aligned}
$$

where $H_{i}^{*}$ is estimated using the least square criteria and $\mathrm{N}$ is the total number of pixels in $_{R_{i}}$. Here we simply use the LBP histograms of $R_{i}$ to approximate $H_{i}^{*}$. In the traditional region competition, the estimation of model parameters is complex. Our algorithm has low computation complexity in this step.

In the second step, $H_{i}$ is fixed and the boundary $\Gamma$ is moved to decrease the energy function in the steepest direction. The motion equation of any point $\vec{v}=(x, y)$ on the boundary can be obtained using the functional derivative of the energy $E$.

$$
\frac{d \vec{v}}{d t}=-\frac{\partial(E)}{\partial \vec{v}}
$$


Referring to the appendix in [1], we can develop the motion equation of $\vec{v}$ as follows:

$$
\frac{d \vec{v}}{d t}=\sum_{k \in\left\{\Gamma_{k} \text { contains } \bar{v}\right\}}-\frac{\mu}{2} K_{k(\bar{v})} \vec{n}_{k(\bar{v})}+S\left(H_{\vec{v}}, H_{k}\right) \vec{n}_{k(\bar{v})},
$$

where $\Gamma_{k}$ represents the boundary of region $R_{i}$, and $\mathrm{k}$ belong to the region set, whose boundaries $\vec{v}$ lies on. $K_{k(\vec{v})}$ is the curvature of $\Gamma_{k}$ at point $\vec{v}$ and $\vec{n}_{k(\vec{v})}$ is unit normal to $\Gamma_{k}$ at point $\vec{v} \cdot \vec{n}_{k(\vec{v})}$ points outwards from the region $R_{i}$.

The equation (5) indicates that the motion of point $\vec{v}$ is controlled by two kinds of forces acting on the boundary, both pointing along the normal. The smoothing force is caused by the first term in (5) and its intensity increases as the curvature increases. It serves to straighten the boundary as possible. The second term represents the statistics force, which tends to be weaker when the local statistics match the region global statistics better.

For example, $\vec{v}$ is on the common boundary of region ${ }_{R_{i}}$ and $R_{R_{j}}$. Since curves $\Gamma_{i}$ and $\Gamma_{j}$ have inverse normal in $\vec{v}$, we have $\overrightarrow{n_{i}}=-\overrightarrow{n_{j}}$ and $K_{i} \overrightarrow{n_{i}}=-K_{j} \overrightarrow{n_{j}}$. Thus the motion equation for $\vec{v}$ can be expressed as:

$$
\frac{d \vec{v}}{d t}=-\mu K_{i(\bar{v})} \vec{n}_{i(\vec{v})}+\left(S\left(H_{\bar{v}}, H_{i}\right)-S\left(H_{\bar{v}}, H_{j}\right)\right) \vec{n}_{i(\vec{v})}
$$

Because the smoothing force has been introduced in [1], we focus on the statistics force here. If $S\left(H_{\vec{v}}, H_{i}\right)>S\left(H_{\vec{v}}, H_{j}\right)$, - i.e., if the histogram of the local patch around $\vec{v}$ matches the histogram of region ${ }_{H_{i}}$ better-then the boundary will move along $\vec{n}_{i(\bar{v})}$. This is the same for the points shared by several regions. In the more adjacent regions cases, the boundary point $\vec{v}$ will be owned by the region with the most similar statistics to the local statistics around $\vec{v}$.

Both the two steps cause the energy to decrease and guarantee it to converge to a local minimum. However, these two steps have not chances to alter the number of the regions. In the second stage, we will decrease the energy by merging adjacent regions.

For example, $R_{i}$ and ${ }_{R_{j}}$ are two neighboring regions, and the pixel numbers and LBP histograms are $n_{i}$ $H_{i}$ and $n_{j} \quad H_{j}$ respectively. Denote $R_{i j}=R_{i} \cup R_{j}$ to the region after merging, denote the LBP histograms of $R_{i j}$, and denote $l_{i j}=\Gamma_{i} \cap \Gamma_{j}$ to be common boundary of ${ }_{R_{i}}$ and $R_{R_{j}}$. Then the change of energy can be expressed as:

$$
\Delta E=-\mu \int_{l_{i j}} d s-\lambda-\sum_{(x, y) \in R_{i j}} S\left(H_{x, y}, H_{i j}\right)+\sum_{(x, y) \in R_{i}} S\left(H_{x, y}, H_{i}\right)+\sum_{(x, y) \in R_{j}} S\left(H_{x, y}, H_{j}\right)
$$

Considering the equation (4), we obtain $H_{i j}=\frac{1}{n_{i}+n_{j}} \sum_{(x, y) \in R_{i j}} H_{(x, y)}, H_{i}=\frac{1}{n_{i}} \sum_{(x, y) \in R_{i}} H_{(x, y)}, H_{j}=\frac{1}{n_{j}} \sum_{(x, y) \in R_{j}} H_{(x, y)}$. Thus equation can be developed as:

$$
\begin{aligned}
\Delta E & =-\mu\left|l_{i j}\right|-\lambda+1 \\
-\sum_{s=1}^{k} & \left(\sum_{(x, y) \in R_{i}}\left(H_{x, y}(s)-H_{i}(s)\right)^{2}+\sum_{(x, y) \in R_{j}}\left(H_{x, y}(s)-H_{j}(s)\right)^{2}-\sum_{(x, y) \in R_{i j}}\left(H_{x, y}(s)-H_{i j}(s)\right)^{2}\right) \\
& =-\mu\left|l_{i j}\right|-\lambda+1-\sum_{s=1}^{k}\left(-n_{i} H_{i}(s)^{2}-n_{j} H_{j}(s)^{2}+\left(n_{i}+n_{j}\right)\left(\frac{n_{i} H_{i}(s)+n_{j} H_{j}(s)}{n_{i}+n_{j}}\right)^{2}\right) \\
& =-\mu\left|l_{i j}\right|-\lambda+1-\sum_{s=1}^{k}\left(\left(\frac{n_{i} n_{j}}{n_{i}+n_{j}}\right)\left(2 H_{i}(s) H_{j}(s)-H_{j}(s)-H_{i}(s)\right)\right)
\end{aligned}
$$


where $\mathrm{s}$ indexes the quantization levels and $\mathrm{k}$ is the number of histogram bins .If $\Delta E<0$, then merging two regions will decrease the energy.

Our region competition embedded with LBP is summarized as follows:

1. Initialize the segmentation, seeds are randomly selected across the image, and all regions, which are not covered by any seeds, are considered to be one region.

2. Fix the boundary and compute the LBP histograms for each region.

3. Fix the LBP histograms for each region and move the boundary to decrease the energy.

4. If the motion of boundary converges, then go to step 5 . If not, go to step 2 .

5. It there still exists background not covered by any seed regions put new seed in the background and go to step 2. If not, go to step 6.

6. If energy can be decreased by merging regions, merge two neighboring regions so that we can obtain the largest energy decrease, and go to step 2. If not, stop.

\section{Experiments and discussions}

We applied our texture segmentation algorithm to several images of composite textures with size $256 \times 256$ pixels and 256 grey levels. Textures from the Brodatz album [10] are used to make up the composite texture images. None of the textures used in our experiment can be discriminated by grey level values alone. We set the radius of circular windows to be 5 pixels, and the size of the LBP/C histogram is 256x8. Figure 2 shows the segmentation results obtained for 3 composite texture images (640x640), which both consist of 4 distinct textures selected from Brodatz album. We randomly set 7 seeds across the images, and we can find the result is promising.
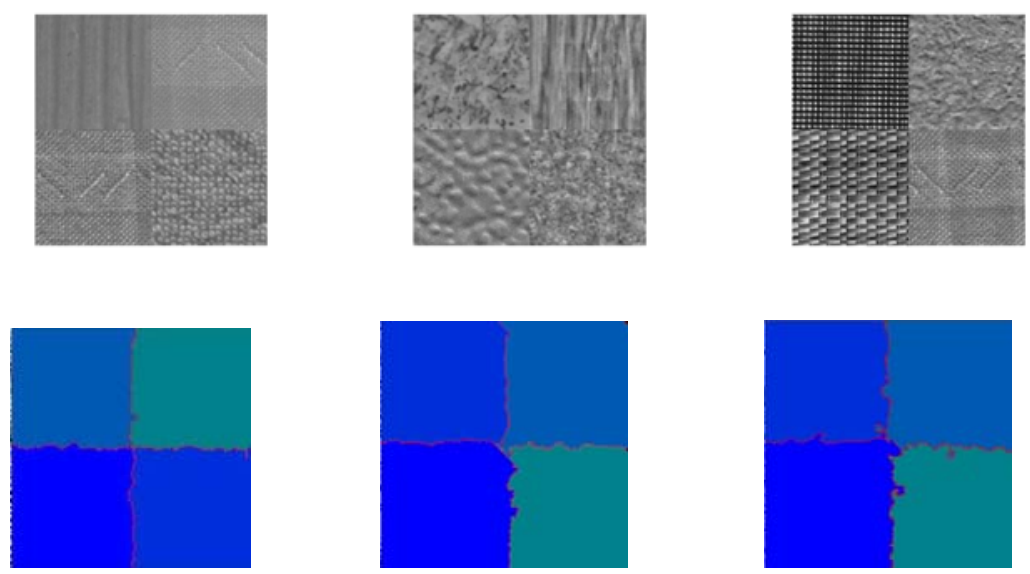

Fig. 2. Three composite texture images and their segmentation results. The first row shows the original image and the second row shows the segmentation results.

\section{Conclusion}

In our paper, we modify the region competition method to segment textures. First, local Binary pattern (LBP) histogram is adopted to capture the texture information. Then, considering the specific goal of texture segmentation, we propose new assumption about region competition and rewrite the energy function based 
on LBP histograms. We also redevelop the two-stage iterative algorithm to make our energy converge to a local minimum. Because of the fast LBP operator and nonparametric histogram model, we can simplify the parameter estimating step, which is the most time consuming step. Besides, LBP high performance for texture characterization helps to make our method more suitable for texture segmentation problem. Experiments show that the performance of our proposed method is promising and obtain a robust and fast segmentation of texture images. We can still extend our work by using LBP/C to describe color texture, and thus can employ our method to segment color textures.

\section{Reference}

[1] S.C. Zhu and A.L. Yuille," Region Competition: Unifying Snakes, Region Growing and Bayes/MDL for Multiband Image Segmentation”, IEEE Trans. Pattern Analysis and Machine Intelligence, vol. 18, no. 9, pp. 884-900, Sept. 1996.

[2] Clark, A.A.; Thomas, B.T., "Evolving image segmentations for the analysis of video sequences, Computer Vision and Pattern Recognition”, CVPR 2001. Proceedings of the 2001 IEEE Computer Society Conference on, Volume: 2, 8-14 Dec. 2001

[3] Ho, S.; Bullitt, E, “Level-set evolution with region competition: automatic 3-D segmentation of brain tumors”. Gerig, G.;Pattern Recognition, Proceedings. 16th International Conference on , Volume: 1, 11-15 Aug. 2002

[4] Ding Siyi, Yang Jie, Xu Qing, “Automatic Segmentation of Three Dimension Medical Image Series using fuzzy region competition", Journal of Biomedical engineering , to appear

[5] T. Ojala, M. Pietikäinen and D. Harwood, "A comparative study of texture measures with classification based on feature distributions”, Pattern Recognition 29, pp. 51-59,1996.

[6]T. Ojala, M. Pietikäinen and J. Nisula, "Determining composition of grain mixtures by texture classification based on feature distributions”, International Journal of Pattern Recognition and Artificial Intelligence 10, pp.73-82, 1996.

[7] T. Ojala, "Multichannel approaches to texture description with feature distributions", Technical Report CAR-TR-846, Center for Automation Research, University of Maryland, 1996.

[8]Harwood D, Ojala T, Pietik"ainen M, Kelman S \& Davis S, “Texture classification by center-symmetric auto-correlation using Kullback discrimination of distributions", Technical report, Computer Vision Laboratory, Center for Automation Research, University of Maryland, College Park, Maryland. CAR-TR678.

[9] Kan-Min Chen, Shu-Yuan Chen, “Color texture segmentation using feature distributions”, Pattern Recognition Letters 23:755-771

[10] P. Brodatz. Textures: A Photographic Album for Artists \& Designers. Dover Publications,

Inc., New York, 1966. 